\title{
LA PROTECCIÓN LEGAL DEL MEDIO AMBIENTE: DESARROLLO SOSTENIBLE Y ACCIONES COLECTIVAS ${ }^{1}$ \\ The Legal Protection of the Environment: Sustainable Development and Collective Actions
}

\author{
Agustín Viguri Perea $^{2}$ \\ Maria Chiara Marullo ${ }^{3}$
}

Fecha de Recepción: 5 de abril de 2016

Fecha de Aceptación: 20 de mayo de 2016

SUMARIO: 1. Introducción; 2. El uso sostenible del medio ambiente; 3. La responsabilidad empresarial; 4. Las acciones de defensa de intereses colectivos: un estudio comparado entre el Derecho español y el Derecho norteamericano; 5. Conclusiones; 6. Referencias bibliográficas

\footnotetext{
${ }^{1}$ El presente trabajo se ha realizado bajo la acción prevista por la Dirección General de Justicia de la Comisión Europea (JUST/2013/ ACTION GRANTS).

${ }^{2}$ Dr. Agustín Viguri Perea, TU (Universidad Jaume I -UJI- de Castellón de la Plana) Licenciado en Derecho norteamericano (University of Pittsburgh, USA) Correo: viguri@uji.es.

${ }^{3}$ Dra. Maria Chiara Marullo, investigadora del proyecto: BUSINESS \& HUMAN RIGHTS CHALLENGES FOR CROSS BORDER LITIGATION IN THE EUROPEAN UNION. ACTION GRANT OF THE EU. Correo: marullo@uji.es
} 


\section{CÓMO SE CITA ESTE ARTíCULO (Normas APA-6)}

Viguri Perea, Agustín, Marullo, Maria Chiara (2016). La protección legal del medio ambiente: desarrollo sostenible y acciones colectivas. Revista Jurídica Mario Alario D’Filippo, VIII (16), pág 135-158.

\section{RESUMEN}

Medio ambiente, empresa y derechos humanos forman parte integrante de un problema social que requiere urgentes respuestas legales. Su resolución debe contemplar estudios de derecho comparado, en el plano del derecho angloamericano y derecho continental. Legislación y jurisprudencia son soportes necesarios para lograr este objetivo, teniendo presente la doctrina internacional imperante en este tópico. Pensamos que la aplicación en materia de daños del principio de la responsabilidad objetiva será esencial para una mejor defensa del medio ambiente y, al mismo tiempo, de la protección de la salud de los ciudadanos. Las acciones de defensa de intereses colectivos son el mecanismo legal adecuado para conseguir estos fines.

\section{PALABRAS CLAVE}

Medio ambiente, empresas, derechos humanos, acciones de clase.

\section{ABSTRACT}

Environmental law, corporations and human rights are part and parcel of a social problem that urgently needs to be remedied. Therefore, the best way of tackling this real crossroad is through comparative law studies encompassing both common law and continental law jurisdictions. Legislation and jurisprudence are the most suitable legal tools so as to achieve this goal, taking also into account the prevailing doctrine in this subject all around the world. From our standpoint, as far as tort law is concerned, the application of strict liability through the principle of "polluter pays" will be the best way of protecting the environment as well as the health of the citizens. Class actions are the most adequate way of reaching these targets.

\section{KEYWORDS}

Environmental law, corporations, human rights, class actions. 


\section{INTRODUCCIÓN}

Como hemos mencionado en el precedente artículo sobre los problemas para la salud y el medio ambiente y la encrucijada de los alimentos transgénicos ${ }^{4}$, en los últimos años la doctrina internacional comenzó a estudiar el tema de los negocios internacionales y los posibles impactos negativos sobre la protección y garantía de los derechos de las personas, la vida, la salud, la seguridad ambiental y alimentaria (i Llobet y Manzano, 2007; Ruiz y Daudí, 2014; Bonfanti, 2012; Cernic, 2013; Bravo, 2013) Una importante práctica jurisprudencial, en particular la que se inició en Estados Unidos y con posterioridad en Europa (Marullo, 2015; Marullo y Zamora, 2016), ha evidenciado la carencia a nivel internacional de normas vinculantes para responsabilizar a las empresas multinacionales por sus acciones $y$, al mismo tiempo, ha puesto de manifiesto que también, en niveles nacionales, hay numerosos obstáculos y barreras que impiden determinar las responsabilidades civiles y penales por los daños ocasionados. ${ }^{5}$

La casuística de violaciones de Derechos Humanos, otros abusos y destrucción de los recursos naturales no se circunscribe a algunos sectores de las actividades empresariales considerados más peligrosos como, por ejemplo, las multinacionales extractivas y minerías en particular, que incurren en violaciones normalmente más visibles y con efectos dañinos a escala, mucho más grandes (Zamora, 2015), sino que abarca desde el sector textil al sector agroalimentario, pasando por muchos otros. Las razones que pueden explicar las dificultades actuales de llevar a cabo juicios sobre dichas conductas son muchas y están íntimamente ligadas a las políticas internas e internacionales de los Estados y su sumisión total a los mercados. ${ }^{6}$

\footnotetext{
${ }^{4}$ En él hemos pretendido destacar la encrucijada de los alimentos transgénicos en Estados Unidos y en Europa y las implicaciones sobre la protección del medio ambiente y la salud de las personas.

${ }^{5}$ Véase Skinner, G., McCorquodale, R., y De Schutter, O. (2013). The Third Pillar: Access to Judicial Remedies for Human Rights Violations by Transnational Business. ICAR/CORE/ECCJ. Recuperado de: http://corporatejustice.org/documents/publications/eccj/ the_third_pillar_-access_to_judicial_remedies_for_human_rights_violation.-1-2.pdf

${ }^{6}$ Recientemente salió a la luz un estudio realizado por la Fundación Global Justice Now sobre las empresas multinacionales más poderosas del planeta. En él se evidencia el poder de control y económico que algunas empresas ejercen sobre los Estados. Como se evidencia en el diario El País:

Para elaborar su lista toma como referencia las 100 mayores entidades económicas del mundo. El ranking identifica a 69 grandes empresas en ese grupo. De ahí toma las 10 compañías más potentes y cruza su facturación con el producto interior bruto de los países. El resultado de la comparación es muy revelador. Estados Unidos, China, Alemania, Japón, Francia y Reino Unido son las entidades económicas más grandes del mundo, seguidas por Italia, Brasil y Canadá. Lo interesante llega justo después. En el grupo de cabeza se clasifica décima Walmart, la mayor cadena comercial del mundo y la primera corporación por ingresos del Fortune Global 500. [...] Si se amplía más la referencia, entre las 20 mayores entidades globales hay tres compañías de origen chino, a saber: el monopolio eléctrico State Grid (14) junto a las petroleras China National Petroleum (15) y Sinopec (16). La clasificación de las 10 compañías más potentes del planeta la completa la petrolera Royal Dutch Shell (18), seguida por Exxon Mobil (21), Volkswagen (22), Toyota (23) y Apple (26). El valor combinado de estas 10 multinacionales es comparable al producto interior bruto de los 180 países más pequeños del planeta, un grupo que incluye a Irlanda, Indonesia, Israel, Colombia, Grecia, Sudáfrica y Vietnam, por citar a las más relevantes. "A este ritmo de crecimiento bastará solo con una generación para que el mundo entero esté dominado por grandes corporaciones". augura Nick Dearden (Pozzi, 2016).

Cuando hablamos de la sumisión de los Estados a los mercados, nos estamos refiriendo al fenómeno que en el mundo anglosajón se conoce como "puertas giratorias", por el cual se produce una circulación sin obstáculos de altos cargos entre los sectores público y privado. Estos movimientos se producen en un sentido y en otro, es decir, desde las instituciones públicas a las empresas transnacionales y viceversa, constituyendo una muestra de lo difusa que es la línea que separa ambos poderes, el político y el económico, y que se basa en la obtención recíproca de privilegios o beneficios.
} 
Con este segundo trabajo intentamos encontrar posibles soluciones a uno de los grandes problemas de nuestro tiempo a través de una nueva visión del derecho al desarrollo. El punto de partida serán los compromisos internacionales adquiridos en la nueva agenda de las Naciones Unidas 2030, cuyo objetivo es avanzar en el tema de las acciones colectivas en defensa de los Derechos Humanos y del medio ambiente.

\section{EL USO SOSTENIBLE DEL MEDIO AMBIENTE}

Resulta ser una verdad incontrovertible que los desafíos planteados en la actualidad tienen que ser resueltos a escala mundial. En otras palabras, deben ser resueltos dentro de un plan de iniciativas globales que sean acordes con la máxima de "vivir bien, respetando los límites ecológicos de nuestro planeta" De la misma forma, la celebración anual del Día Mundial del Medio Ambiente establecido por la Asamblea General de las Naciones Unidas en el año 1972, que coincidió con la Primera Cumbre Mundial sobre Medio Ambiente y la Conferencia de Estocolmo sobre Medio Ambiente Humano, tiene los efectos de servir de recordatorio a países y ciudadanos al alimón del reto mundial que supone la defensa de nuestro entorno en el contexto tecnológico actual. Para ello se deberán continuar los acuerdos adoptados en la Conferencia Río + 20, conocida también como la Cuarta Cumbre de la Tierra, organizada por las Naciones Unidas, sobre Desarrollo Sostenible, que tuvo lugar en 2012, cuyo eje central fue la defensa de los derechos humanos en materia de medio ambiente. En la Segunda Cumbre, celebrada en 1992, también en la ciudad de Río de Janeiro, ya quedó proclamado que los seres humanos constituyen el centro de las preocupaciones relacionadas con el desarrollo sostenible.

La tesis ya universal del desarrollo sostenible (sustainable development), aceptada por la mayoría de los sistemas legales mundiales, apelando a la doctrina de la conjugación del crecimiento económico con la protección del medio ambiente, afirma que la tecnología es un producto de la inteligencia humana. Así, más que un cuestionamiento directo al desarrollo tecnológico, el verdadero reto está en "reducir a sus justos límites los efectos negativos que un uso desmesurado, descontrolado y anárquico, acarrearía a toda la sociedad" (Tribunal Supremo, Sala Penal, Núm. 3.851.-Sentencia, 1990).

Esta tesis está presente en los 17 Objetivos de Desarrollo Sostenible, adoptados en una histórica cumbre en septiembre de 2015 en Nueva York por 193 Estados ${ }^{7}$, que tratan de abordar todas las necesidades y el acceso a bienes, servicios y derechos de las personas, indispensables para promover el respeto de la dignidad y el pleno desarrollo de las sociedades. Podemos decir que la nueva Agenda de las Naciones Unidas 2030 para el desarrollo sostenible ${ }^{8}$ atiende también a una nueva visión del desarrollo (Sachs, 2015;

\footnotetext{
${ }^{7}$ Mediante la Resolución de la Asamblea General de 25 de septiembre de 2015, Transformar nuestro mundo: la Agenda 2030 para el Desarrollo Sostenible, Doc. A/RES/70/1, de 21.10.2015.

${ }^{8}$ Las metas de esta Nueva Agenda puede consultarse en Asamblea General de las Naciones Unidas. (2015c). La Agenda de Desarrollo Sostenible. Recuperado de: http://www.un.org/sustainabledevelopment/es/la-agenda-de-desarrollo-sostenible/ Oficialmente la agenda entró en vigor el 1 de enero de 2016. Siguiendo a Cortina (2006): el concepto que se está manejando no está restringido al
} 
Čiegis y Ramanauskienè [2009]) que tiene como fin el crear sociedades que garanticen, a través de la priorización del acceso a la justicia, la plena satisfacción de sus miembros (Dallo, 2016; Sánchez, 2016; Guimarães, 2015; Mendizábal, 2013; Dernbach y Cheever, 2015).

Estos objetivos son normas de soft law internacional pues están diseñados conforme al desarrollo de las normas internacionales en estas materias. Es evidente que la intención de la nueva agenda es crear una conexión con dichas reglas y las normas de hard law en tema de protección y garantía de los Derechos Humanos y del medio ambiente (Kim,2016) Este punto de contacto se materializa, sobre todo, cuando se reconoce la necesidad de construir sociedades inclusivas, pacíficas y sostenibles, teniendo como prioridad el acceso a una justicia más equitativa, efectiva y a todos los niveles ${ }^{9}$ Así, en la nueva Agenda se declara que es posible lograr el uso sostenible del ambiente, pero sin acrecentar necesariamente las diferencias entre el Norte y el Sur.

Podemos ver cómo, en virtud de los compromisos internacionales, los Estados se comprometieron inter alia a la protección de la dignidad, a eliminar todas formas de discriminación, a garantizar la equidad y el estado de derecho y, finalmente, a proteger el medio ambiente. Por tanto, estarían obligados a asegurar el pleno ejercicio de los derechos fundamentales a través su acceso universal, por lo menos a nivel básico, así como el acceso a los recursos cuando tales derechos sean vulnerados por el sector público o el sector privados (Marullo y Pallares, 2016) En la misma línea, la nueva agenda establece pautas para involucrar en el proceso de desarrollo sostenible a todos los agentes que pudieran estar implicados de forma activa y pasiva en la creación de sociedades pacíficas e inclusivas, como puede ser el caso de las empresas del sector privado, entre ellas las multinacionales. Se entiende entonces a las empresas privadas como entidades que, con su participación, son capaces de fomentar el desarrollo humano para hacer que la tutela de los derechos de las personas sea efectiva. Como uno de los protagonistas más importantes en la adquisición de estos compromisos, sus acciones tienen repercusiones tanto en el sentido social, buscando preservar los derechos de los grupos y las clases sociales, como en el sentido individual procurando hacer efectivo el derecho al libre desarrollo de la personalidad.

Con respecto a esto último, en 1987 la Comisión mundial sobre Ambiente y Desarrollo dejó un mensaje claro en su informe "Nuestro futuro común": el crecimiento económico

progreso económico sino más bien se vincula al concepto de desarrollo humano, donde los protagonistas de este proceso llegan a ser las personas, basado en pilares fundamentales como la dignidad, personas, planeta, justicia y asociación. Un desarrollo que sea sostenible, por tanto, tiene que contemplar tres diferentes dimensiones indivisibles e interconectadas, económica, social, y ambiental. En relación a las tres dimensiones del desarrollo se puede consultar Lehtonen (2004), Krajnc y Glavic (2005), Redclift (1991).

${ }^{9}$ El Objetivo 16 de los 17 objetivos para transformar nuestro mundo incluye: Promover sociedades pacíficas e inclusivas para el desarrollo sostenible, facilitar el acceso a la justicia para todos y crear instituciones eficaces, responsables e inclusivas a todos los niveles. Para más información: Naciones Unidas. (s.f). Objetivo 16: Promover sociedades, justas, pacíficas e inclusivas. Recuperado de: http://www.un.org/sustainabledevelopment/es/peace-justice/ Véase también Foundation for Democracy and Sustainable Development. (s.f). UN Sustainable Development Goal (SDG) 16-importance of participatory institutions \& policymaking. Recuperado de: http://www.fdsd.org/ideas/sustainable-development-goal-sdg-16-democratic-institutions/y Mesa, M. (2016). El Objetivo n. 016 de Desarrollo Sostenible: paz, seguridad y gobernanza. Temas para el debate, (254), 37-40. 
no puede alejarse de un ambiente sostenible y de un desarrollo social a largo plazo, y estableció, así, el desarrollo sostenible como un marco válido para integrar el desarrollo económico y la protección del medio ambiente y la estabilidad social. De esta forma elevó el desarrollo sostenible a la categoría de la ética global que se fundamentaría en los principios de justicia, responsabilidad y solidaridad. Desde ese momento se auspicia una remodelación del sistema internacional existente abriendo la puerta a nuevos actores a participar en el proceso de desarrollo. De hecho, sin una ética global, los diferentes actores no podrían llegar a ser participantes activos y constructivos en el proceso mundial de desarrollo sostenible. A partir de esta toma de consciencia acerca de las actividades empresariales, el debate internacional se está interrogando sobre la posible incorporación de las dimensiones éticas, ambientales y sociales del desarrollo en los negocios, y cómo el sector privado podría participar de forma activa en el proceso de desarrollo sostenible, pero, al mismo tiempo, sobre la manera en que este podría rendir cuenta por sus actividades ilícitas. Como explica Marzá (2009) las empresas del sector privado pueden ser entendidas como instituciones que, capaces de fomentar o no el desarrollo humano, tienen que responsabilizarse por sus actuaciones. Por tanto, los Estados deberían implementar los mecanismos existentes o crear nuevos para que esos agentes puedan rendir cuentas por los daños causados en contra de los Derechos Humanos o del medio ambiente.

Llegando a este punto nuestra pregunta es: ¿Cómo es posible hablar de un acceso equitativo a la justicia tanto en el ámbito internacional como en el nacional, en sistemas legales donde los individuos no tienen las mismas condiciones que otros actores del sector privado y público, en particular, en el caso de las empresas multinacionales, o cuando los mismos ordenamientos jurídicos excedan sus posibilidades financieras o de conocimientos? En otras palabras, ¿cómo podemos superar estos obstáculos que nos demuestran que nos enfrentamos a una desigualdad judicial (unequal legal battles)? ${ }^{10}$

Para superar estos obstáculos partimos, desde la perspectiva del derecho civil, de un silogismo, esto es, de dos proposiciones que nos conducen a un resultado final. Así, resulta incontrovertible el hecho de que los contratos, en la actualidad, se caracterizan por realizarse generalmente en masa, por tanto, los daños que puedan surgir serán también en serie, esto es, masivos. La solución más eficaz a este problema es, a nuestro juicio, la aplicación de las acciones de clase.

\section{LA RESPONSABILIDAD EMPRESARIAL}

Las empresas multinacionales tienen diferentes códigos de conducta y estrategias comerciales en lo que se refiere a las actuaciones que llevan a cabo en los países menos desarrollados con respecto a los países del primer mundo. No obstante, cuando hablamos de responsabilidad empresarial por graves violaciones a los derechos humanos

10 Sobre este tema, véase el caso Texaco-Chevron en Ecuador en Pigrau, A. (2014). The Texaco-Chevron case in Ecuador: Law and justice in the age of globalization. Revista Catalana de Dret Ambiental, 5(1), 1-44. 
y ambientales nos encontramos frente a un ángulo muerto en el derecho internacional y nacional y con ello no podemos garantizar los remedios judiciales para que las víctimas puedan ser indemnizadas por lo sufrido (Solanes, 2016).

Pensamos que es representativo de este aserto, entre los múltiples ejemplos que nos vienen a la memoria, el desastre de Bhopal (India), conocido como el "penoso asunto de la gran cámara de gas", causado por la compañía norteamericana Union Carbide, actualmente, en control de Dow Chemical, que provocó la muerte de 22.000 personas. Una tragedia que, después de trascurridos 32 años, no ha encontrado todavía solución en los tribunales. Las víctimas siguen reclamando 8.100 millones de dólares de indemnización por negligencia tras el escape de 40 toneladas de gas con escasas opciones de conseguirlo. También cabe mencionar el caso del derrumbe de un edificio textil en Bangladesh. En abril de 2013 el edificio Rana Plaza, en Bangladesh, se vino abajo causando la muerte de más de mil persona (Laronze y de Quénaudon, 2013; Cyran, 2013; de Miguel Beriain, 2013; Lund-Thomsen y Lindgreen, 2014; Gomes, 2013; Evans, 2014) Una prueba más de que la economía asume el desarrollo tecnológico en función de la rentabilidad, sin prestar atención a las eventuales consecuencias negativas para el ser humano, y de que el avance de la ciencia y de la técnica no equivale, necesariamente, al avance de la humanidad.

Para el cumplimiento del derecho de daños causados en serie, categoría que engloba en su tratamiento jurídico los accidentes colectivos (se pueden citar, supuestos de accidentes aéreos o derivados de emanaciones de substancias tóxicas con altos porcentajes de muertes o lesiones, como en el caso Bhopal o en aquellos otros relacionados, a título de ejemplo, con el asbesto) se encuentran mejores soluciones procesales, en nuestra opinión, en el marco de aplicación de las acciones en defensa de intereses colectivos, esto es, de las acciones de clase. Fue a finales de la década de los 80, cuando esta opción cobró extraordinaria importancia en el derecho norteamericano como derecho de daños derivados de desastres masivos.

Ahora bien, uno de los grandes retos a los que debemos enfrentarnos para una adecuada globalización del derecho que conduzca a la defensa de los derechos humanos, así como del medio ambiente, es el reconocimiento de las necesidades de los sujetos afectados. En un contexto legal en el que se compra y se vende en serie, esto es, se contrata en masa (mass contracts) y se producen daños masivos, es decir, en masa (mass torts), es necesario introducir fórmulas de acceso a la justicia que apelen a remedios colectivos.

Estaríamos hablando, ni más ni menos, que de la implantación de las acciones de clase (class actions), es decir, de las acciones en defensa de intereses colectivos, en el marco del derecho continental, en general y, en España, en particular. Su origen se remonta al Bill of Peace británico que, como recurso de prevención de múltiples litigios, procuraba reunir un importante número de cuestiones de hecho o de derecho para decidir sus 
posibles soluciones, mediante un procedimiento basado en la equidad (equitable procedure). Desde nuestra perspectiva, en términos de garantía de acceso a la justicia, este mecanismo sería capaz de reducir los costes de los juicios y garantizar también una adecuada tutela de las víctimas de ilícitos perpetrados por empresas multinacionales, solventando también otros obstáculos jurisdiccionales y de procedimiento.

Por tanto, desde una visión civilista comparatista aconsejamos su implantación, a sabiendas de que diferentes ordenamientos jurídicos europeos han optado por la defensa individualizada, generalizada debido a la influencia del Derecho romano. En este sentido debemos mencionar el cambio que se ha producido en Francia a través de la aprobación de la Ley núm. 2016-1547, el 18 de noviembre de 2016, de modernización de la justicia del siglo XXI ${ }^{11}$ Interesa destacar el Título V del Capítulo Primero, relativo a las acciones colectivas ante el Poder Judicial, que regula las acciones planteadas sobre el medio ambiente, la salud pública y la regulación de los datos y ficheros informáticos. Estas reglas siguen la normativa prevista en el Código de Procedimiento Civil. La Sección Primera tiene como objeto las acciones colectivas, que son presentadas por las asociaciones, relativas a la cesación o reparación de los perjuicios sufridos por los afectados. Se requiere que tales asociaciones deban estar constituidas cinco años, al menos, con anterioridad a la presentación de la demanda. Las mencionadas acciones de clase deben ser entabladas una vez expirado el termino de cuatros meses, necesarios para la presentación de la defensa.

Por eso, estamos convencidos que se debería abandonar el paradigma de la litis individual, que caracteriza a la práctica totalidad de los ordenamientos jurídicos europeos, como en el caso de Alemania. En cambio, en el Derecho angloamericano su aplicación ha sido materializada con éxito en múltiples ocasiones. Por ejemplo, en relación al asbesto como en el litigio Manville (Estado de Nueva York), a las prótesis mamarias de silicona fabricadas por la multinacional Dow Chemical (concentradas en el Estado de Alabama), a los efectos nocivos del tabaco (Estado de Florida) y, más recientemente, respecto del fraude en las emisiones de automóviles (Estado de California).

\section{LAS ACCIONES DE DEFENSA DE INTERESES COLECTIVOS: UN ESTUDIO COMPARADO ENTRE EL DERECHO ESPAÑOL Y EL DERECHO NORTEAMERICANO}

Introducidas en la práctica jurídica de los Estados Unidos a partir de los años 60, gozan de una gran tradición tras haber conseguido eliminar importantes barreras jurisdiccionales y de procedimiento ${ }^{12}$ En nuestro análisis, siguiendo el método del caso, recogemos uno de los temas de mayor y más candente actualidad a nivel mundial, como es el que afecta

\footnotetext{
${ }^{11}$ Asamblea Nacional. (18 de noviembre del 2016). Para modernizar la justicia del Siglo XXI. [Ley 2016-1547]. Recuperado de: https:// www.legifrance.gouv.fr/eli/loi/2016/11/18/JUSX1515639L/jo

${ }^{12}$ Véase Corte Suprema de California. (mayo 10 de 1971). Vasquez v. Superior Court, 484 P.2d 964. Recuperado de: https://www. courtlistener.com/opinion/1185687/vasquez-v-superior-court/ y A. Azagra y Marian Gili. (2005). Guia InDret de jurisprudencia sobre responsabilidad civil por daños del amianto. Indret (2).Recuperado de: http://www.asviamie.org/documentos/condenas_amianto.pdf
} 
a los damnificados por el uso del amianto. Uno de los primeros precedentes fue Borel $v$. Fibreboard, en el que se decidió que las industrias relacionadas con el amianto debían indemnizar a las víctimas. En In re Joint Eastern ${ }^{13}$, los hechos se sitúan en plena década de los años 70. Johns Manville Corp., la más importante de las compañías fabricantes de amianto del mundo, fue demandada por miles de personas afectadas de asbestosis ${ }^{14} \mathrm{Se}$ entablaron cientos de demandas, consiguiendo los damnificados una serie significativa de veredictos, por medio de jurados, a su favor, tras haber podido probar los siguientes extremos:

a) Que la compañía había vendido un producto peligroso.

b) Que la empresa conocía en el momento de la venta que el producto era defectuoso, rechazándose la defensa de la compañía amparada en el argumento conocido como "state of art" (o sea, en el "estado actual de la tecnología").

c) Que los afectados habían contraído esta penosa enfermedad por su exposición al asbesto en el cumplimiento de sus funciones.

Las indemnizaciones que tuvo que satisfacer la compañía la llevaron a la quiebra en 1982. En 1988, Manville se reconvirtió y empezó a concentrarse en otros negocios pertenecientes a la empresa, viéndose en la necesidad de crear un trust, que sirviera para compensar a las víctimas de la enfermedad, al que tenía asignada una parte elevada de sus beneficios netos, bonos y acciones. En apenas un par de años, la compañía se vio incapaz, nuevamente, de resarcir a todos los afectados de asbestosis. El panorama se ensombrecía, además, por el hecho de que dicha enfermedad tiene un período de incubación que puede llegar a los 50 años, en casos extremos, lo que motivó que la aparición de nuevas demandas pudiera entrar en una espiral sin fin. En 1990, el cálculo estimativo de posibles reclamaciones alcanzaba cifras escalofriantes, en torno a las 300.000, cuando la compañía contaba ya, en esas fechas, con unas deudas que sobrepasaban los 4 billones de dólares.

En suma, se requería un replanteamiento del problema, por lo que se optó por la propuesta de arreglo (settlement) de la defensa, consistente en la certificación de una class action, con base en la Regla 23 (b) (1) del Federal Rules of Civil Procedure ${ }^{15}$, que sirviera para agrupar a todos los demandantes presentes y futuros, bajo las siguientes condiciones, a saber:

\footnotetext{
${ }^{13}$ U.S. District Court for the Southern District of New York. (27 de junio de 1991). In re Joint Eastern and Southern District Asbestos Litigation. 129 B.R. 710 (S.D.N.Y. 1991) Recuperado de: http://law.justia.com/cases/federal/district-courts/BR/129/710/1871302/

${ }^{14}$ Enfermedad pulmonar que habían contraído al estar expuestas a dicho mineral mientras trabajaban en astilleros y empresas de la construcción.

${ }^{15}$ Este modelo procesal norteamericano que se regula en la Regla 23 a), en el marco de las Reglas Federales de Procedimiento Civil (Federal Rules of Civil Procedure) del año 1938, que fueron objeto de enmienda, en el año 1966 por el Comité Consultivo sobre Reglas Civiles (Advisory Committee on Civil Rules), constituye una técnica de demanda colectiva para el control inhibitorio o sancionador del comportamiento de una empresa y, como tal, configura un instrumento legal confiable a la iniciativa de las asociaciones adecuadamente representativas de grupos de consumidores. Las posteriores revisiones facilitan su comprensión, y amplían algunos plazos en cuanto a su aplicación.
} 
a) Cada afectado recibiría, inicialmente, sólo el $45 \%$ del montante de su reclamación, y el resto de la cantidad quedaba pendiente hasta que los demás actores hubieran, asimismo, obtenido dicha suma.

b) Una parte importante de los ingresos que fueran afluyendo a favor del demandado (trust) quedarían reservados para posibles futuros demandantes, eliminándose de esta manera el riesgo de insolvencia de la compañía.

c) Aunque no es legítima la supresión del derecho constitucional, que asiste a todo ciudadano norteamericano a defender sus intereses ante un jurado, la compensación que se pudiera lograr por esta vía legal nunca podría sobrepasar la cantidad estipulada en el acuerdo, ya que ello implicaría poner en peligro las compensaciones del resto de los afectados.

d) Las personas cuyos daños revistieran una mayor gravedad, obviamente, deberían ser indemnizadas, en primer lugar, es decir, con carácter preferencial sobre el resto de los damnificados por el asbesto.

e) Por último, el carácter vinculante del procedimiento a seguir no podía ponerse en tela de juicio, de ahí la inexistencia de un derecho de exclusión. En otros pleitos, incluso se ha llegado a admitir (caso Dow Chemical).

El juez Weinstein aprobó la certificación de la clase, así como el arreglo descrito, a través de una serie de argumentaciones, que constan ya en los anales de la jurisprudencia norteamericana, entre los que destacamos los siguientes:

a) Suitability: La Regla 23, que establece la nota de obligatoriedad de la acción de clase, basada en la conveniencia de asignar un fondo limitado para el pago de posibles demandantes. Era la decisión más eficaz para afrontar el problema ante la precariedad de recursos del "trust".

b) Extreme need: Ante un caso de extrema necesidad como el presente, lo más recomendable era la evitación de la repetición de litigios, y de determinaciones fácticas como las que, inicialmente, se hicieron en las demandas tramitadas por separado. En consecuencia, se imponía el fortalecimiento del arreglo mediante la vinculación de los reclamantes ausentes.

c) Statutory requirements: Los requisitos de la Regla 23 (a) para el otorgamiento de la certificación a la clase se estimó que se habían cumplido. Para lo cual, el Juez procedió a examinar cada uno de ellos mediante el análisis que recogemos a continuación:

- The numerosity requirement: La existencia de cientos de miles de presentes y futuros litigantes así lo atestiguaba.

- The commonality requirement: Todos los demandantes tenían varios vínculos en común, puesto que, sobre todo, se cernía sobre todos ellos el interrogante en torno a la posibilidad de que el demandado pudiera satisfacer íntegramente la compensación debida a los afectados.

- The tipicality requirement: Como consecuencia del aserto anterior cada uno de los miembros del colectivo corría el riesgo de no poder materializar sus demandas 
ante la posible insolvencia del demandado.

- The adequacy of representation: Los demandantes estaban representados por los más notables y expertos pleiteadores en la especialidad de amianto de toda la nación. Desafortunadamente, ello quedó también reflejado en las altas minutas a satisfacer por su defensa.

d) Requirements for (b) (1) class: El requisito adicional de la Regla 23 (b) (1) (b), que defiende que los intereses de los miembros ausentes de la clase no sean perjudicados por acciones individuales, no parecía ofrecer tampoco obstáculo alguno.

e) Class certified: El grupo de damnificados fue certificado dentro de la categoría (b) (1). Ello suponía que ninguno de los afectados podría percibir como compensación una cantidad superior a la estipulada en el arreglo concertado.

f) Settlement approved: El Juez aprobó el acuerdo ya que era perfectamente razonable, abrazando un criterio objetivo, a tenor de las circunstancias existentes en el controvertido caso.

En el ámbito normativo corresponde a la Agencia de Protección Ambiental del gobierno federal de los Estados Unidos (Environmental Protection Agency-EPA-) la regulación del amianto. Mediante la aplicación del Código de Regulaciones Federales (Code of Federal Regulations -CFR-), que se contempla en el Título 40, Sección 763, se prohibió la producción, la importación, el procesamiento y la comercialización de los productos relacionados con el asbesto, a partir del año 1989. No obstante, tras la sentencia del caso Corrosion Proof Fittings et. al. v. EPA ${ }^{16}$ el alcance del precepto legal se limitó a restringir su uso a fieltros para suelos, papeles aislantes y nuevos posibles usos, permitiendo su importación en pequeñas cantidades para consumo personal, o envíos a otros países sin embalaje o procesamiento dentro del territorio de Estados Unidos.

Participamos del criterio de que dando carta de naturaleza en el derecho procesal español a estas acciones judiciales, propias del derecho estadounidense y, en general de los países integrantes de la Commonwealth, se permitiría al Ministerio Público poder ejercitar ante los órganos jurisdiccionales competentes, por razón de la materia, una acción de condena de las infracciones de los derechos de los consumidores, incluso de naturaleza económica, que afectasen a un colectivo indeterminado. En el contexto actual en el que se inscriben las sentencias por daños derivados de la exposición al amianto, que causan el fallecimiento o graves enfermedades (relativas no solamente a la asbestosis, sino que generan un amplio radio de acción, como en el caso del carcinoma primitivo de bronquio o pulmón, el mesotelioma pleural o peritoneal, o cáncer de laringe) en nuestro país resulta de evidente y urgente necesidad la búsqueda de soluciones al respecto, que puedan ayudar a resolver los conflictos por la falta o nula aplicación de medidas preventivas de las empresas o la ausencia de diligencia de las mismas en sus actuaciones para evitar

${ }^{16}$ U.S. Court of Appeals for the Fifth Circuit. Corrosion Proof Fittings, et al., Petitioners, v. the Environmental Protection Agency and William K. Reilly,administrator, Respondents, 947 F.2d 1201 (5th Cir. 1991) Recuperado de: http://law.justia.com/cases/federal/ appellate-courts/F2/947/1201/153685/ 
el daño producido. Todo ello, mediante el recurso a las subsiguientes acciones, verbi gratia, en lo que afecta a la responsabilidad civil del empresario derivada de enfermedad profesional, con base en el artículo 1902 del Código Civil Español, a las establecidas en el artículo 1101 respecto de dolo, negligencia o morosidad en las actuaciones, así como los plazos de prescripción recogidos en el artículo $1974^{17}$

El Tribunal Superior de Justicia del País Vasco (Sala de lo Social), en su sentencia $25 / 10 / 2005$, en relación a la responsabilidad civil, estimó que la empresa no facilitó a los operarios medios de protección personal en el desempeño de su trabajo (como el uso de mascarillas). El Instituto Nacional de la Seguridad Social (INSS) ya había dictado una resolución, en el año 2002 declarando al trabajador afecto de incapacidad permanente total por enfermedad profesional. EI TSJPV en su decisión hizo una clara referencia a la culpa que recoge el CC en su artículo 1104, respecto de la diligencia debida que debe prestarse en el cumplimiento de las obligaciones, y que es la exigible o correspondiente a un buen padre de familia (parámetro de tipo medio o normal), que responde al concepto de una persona sujeto de derecho dentro de la clásica corriente romana. Esto es, un empresario vigilante o empleador muy cuidadoso, de acuerdo con su propia entidad. No se trata de aquella culpa excesiva o lata, ni tampoco de la levísima, sino la que es más acorde, en conformidad con la naturaleza de cada obligación, teniendo en cuenta las circunstancias de la persona, del desempeño de la actividad laboral, así como del tiempo y del lugar de ejecución.

Recientemente, el TSJPV se pronunció desestimando el recurso de IZAR Construcciones Navales, en el sentido de reducir en un $50 \%$ la indemnización por el hábito de fumar del fallecido por un carcinoma pulmonar derivado de su exposición al amianto, sin lograr su acreditación, y otorgó 216.956 euros a la familia de un trabajador. La sentencia recoge el criterio del Tribunal Supremo por el que no procede realizar deducción de la indemnización por el daño moral de los ingresos percibidos de la Seguridad Social, como pensiones de viudedad y orfandad, ya que no guardan la necesaria homogeneidad respecto a la indemnización civil.

De ahí, llegamos a la conclusión de que el mero cumplimiento de las reglamentaciones administrativas o laborales no debiera ser causa suficiente para excluir la concurrencia de culpa o la ruptura del nexo causal entre la conducta del empresario y el resultado lesivo. Ello es así, en cuanto se ha producido un daño que, como hemos señalado, puede abarcar un extenso período de tiempo (supra), tras haber estado expuesto el operario en la realización de su trabajo, bien directa o indirectamente, a la inhalación de fibras de asbestos.

\footnotetext{
${ }^{17}$ Verbi gratia, siendo el dies a quo el de la fecha del diagnóstico definitivo de la enfermedad, en este caso, la de la resolución firme de la declaración de la minusvalía, como dice la STSJ Galicia 17-11-2004, y entre esta fecha y la de la presentación de la demanda no debía de haber transcurrido más de 1 año.
} 
En fecha reciente se ha producido un importante avance en materia de infracción de las normas de prevención de riesgos laborales al haberse extendido la responsabilidad empresarial a la empresa sucesora en la actividad, debido a la fusión por absorción de dos sociedades anónimas, Rocalla S.A. por Uralita S.A. En este sentido, nuestro Tribunal Supremo, en la sentencia de 23-3-2015, se ampara, de acuerdo con la primacía de la jurisprudencia comunitaria, en la decisión del Tribunal de Justicia de la Unión Europea, sentencia de 5-3-2015, resolviendo procedimiento prejudicial en torno a la aplicación de la Directiva 78/855/CEE (por obvias razones temporales), actualmente derogada y sustituida por la Directiva 2011/35/UE, pero que mantiene idéntica literalidad en los preceptos que el TJUE interpreta.

En lo que afecta a la determinación de las cuantías relativas a las indemnizaciones, el legislador ha optado de un modo orientativo por aplicar el baremo dentro del sistema que contempla la Ley sobre Responsabilidad Civil y Seguro en la Circulación de Vehículos a Motor ${ }^{18}$ En lo que se refiere a la falta de unificación de la doctrina aplicable, cabe poner de relieve que se están provocando perjuicios irreversibles en sede de reclamaciones judiciales, ya que las sentencias alcanzan, por un lado, fallos bien distintos, además de prolongarse, por otro lado, excesivamente en el tiempo. Somos de la opinión de que esto supone un derroche para las arcas públicas, sin hacer hincapié en el colapso que sufren nuestros tribunales ordinarios de justicia. A título de ejemplo podemos mencionar el caso del Tribunal Supremo en su Sala de lo Social ${ }^{19}$ De ahí nuestra firme defensa de los acuerdos entre las distintas partes en litigio, esto es, de las víctimas del amianto y de las empresas afectadas. Precisamente, la mayor indemnización, alcanzada en julio de 2009 (Garrido, 20009), tuvo su origen a través de esta vía legal, entre 71 trabajadores frente a su empresa (Unión Naval de Levante), mediante una acción penal. ${ }^{20}$

Aunque puede admitirse, en un principio, que las consecuencias ciertas del uso del amianto eran un tanto desconocidas, debido a que el "estado de la ciencia" no contribuía de un modo preciso a aportar soluciones al respecto, no puede pasar tampoco desapercibido el hecho de que las empresas (sobre todo, en el campo de la construcción) utilizaron un producto que sabían podía ser "previsiblemente peligroso" Si bien la primera regulación legal a la exposición por inhalación de sus fibras data en nuestro país del año 1961, fecha en la que se incluyó la asbestosis como enfermedad profesional, no obstante, hasta 2001, no quedó prohibida su comercialización (el crisolito o amianto blanco fue la última modalidad que se seguía utilizándose en España). Las primeras demandas se entablaron a partir de la década de los 90, y su aumento ha sido progresivo, incluyendo reclamaciones

\footnotetext{
${ }^{18}$ En virtud del Real Decreto Legislativo, de 8/2004, de 29 de octubre, se aprobó el Texto Refundido.

${ }^{19}$ Cabe recordar que es la jurisdicción con mayor número de demandas, y ha tenido que dirimir, en algunas ocasiones, hasta 12 sentencias en el escaso período de tiempo de 2 meses, así aconteció en el intervalo transcurrido entre los meses de noviembre y diciembre de 2015

${ }^{20}$ Jurisdicción a la que se ha recurrido para fijar la responsabilidad por fallecimiento. Máxime teniendo en cuenta que, asimismo, según recientes estudios del Instituto de Salud Carlos III, el Consorcio de Investigación Biomédica de Epidemiología y Salud Pública, así como del Ministerio de Sanidad, Servicios Sociales e Igualdad de España, hasta el año 2040, se seguirán produciendo muertes por exposición al amianto en nuestro país.
} 
de vecinos y familiares que residían en las áreas próximas a las factorías, al tratarse del foco originario de dicha enfermedad, causante de problemas físicos, psíquicos y morales a los afectados.

Respecto a los daños morales, la sentencia pionera en el género fue la proveniente del Juzgado de 1a Instancia no 46 de Madrid (20-7-2010), condenando a Uralita al abono de 4 millones de euros a 45 vecinos que habitaban en las inmediaciones de esta fábrica. Interesa destacar de una manera especial que se admitieron como medios de transmisión del asbesto, causantes de sus enfermedades respiratorias (procedentes de las emisiones de la empresa, en forma de polvo), la manipulación de las ropas de los trabajadores por sus familiares en los respectivos domicilios, en conjunción con la contaminación derivada de la degradación de los depósitos de residuos de la propia actividad industrial de la compañía. A pesar del hecho de que fue revocada por la Audiencia Provincial de Madrid, dos años más tarde, al estimar la prescripción de las acciones, fue recurrida ante el Tribunal Supremo (Sala Civil), hallándose, en el momento presente, pendiente aún de resolución.

EI TS, en un principio, se mostró favorable a aplicar la doctrina de la culpa contractual empresarial de una manera bastante restrictiva, desde una perspectiva, más bien, clásica. Posteriormente, hizo hincapié en la defensa de la salud de los trabajadores, a través de un auténtico giro jurisprudencial de corte copernicano, lo que puede comprobarse en los distintos fundamentos jurídicos de las sentencias de 30-11-1997 y 30 de junio de 2010, respectivamente. A la parte demandada se le exigió que aportara las medidas necesarias tendentes a prevenir o evitar el riesgo de las enfermedades que pudieran contraerse por el contacto, mediante, por ejemplo, la dotación de equipos de protección que sirvieran para prevenirlas.

Empero, existen algunos tribunales que han optado por reducir la indemnización por daños y perjuicios aplicando la doctrina de compensación de culpas, al establecer diferentes causas de la enfermedad que provocó el fallecimiento de un trabajador. En esta dirección se manifestó el Tribunal Superior de Justicia del País Vasco (Sala de lo Social) en su sentencia 15/15/2014 (Recurso 1263/2014), al estimar en la primera de las mismas la atribución de la exposición al amianto a la empresa, pero en la segunda consideró que el hecho de ser fumador era relevante en orden a reducir en un $25 \%$ la compensación final. Al difunto le fue diagnosticado EPOC (enfermedad pulmonar obstructiva crónica), asbestosis en 2007 y cáncer de pulmón en marzo de 2012 (un mes anterior a su defunción). Se determinó en la decisión su consumo de tabaco (50 paquetes anuales) hasta que dejó de fumar "cuando menos para finales de hasta 1999", más de 12 años antes de su muerte.

La Unión Europea no prohibió expresamente su comercialización hasta la aprobación de la Directiva 1999/77/CE, que fue objeto de transposición, por Orden del Ministerio 
de la Presidencia, de 7-12-2001, por la que se concedió a los Estados miembro un plazo (finales de 2005) para introducir su regulación. La protección de los trabajadores contra los riesgos relacionados con la exposición al amianto durante el trabajo queda reflejada en la Directiva 83/477/CEE, modificada por la Directiva 91/382/CEE. En términos más generales, cabe mencionar también la Directiva 98/24/CE, sobre la protección de la salud y la seguridad de los trabajadores contra los riesgos relacionados con los agentes químicos durante el trabajo, modificada, a su vez, por la Directiva 2014/27/UE, con los posteriores actos modificativos, en virtud de las Directivas 2003/18/CE, y 2007/30/CE. ${ }^{21}$

El derecho de consumo, en términos generales, como disciplina propia del derecho privado, en concreto, incluida dentro del campo del derecho civil, ha adquirido un gran auge en estos últimos tiempos, tanto en el derecho europeo como en la esfera de la familia del derecho anglosajón. Sirve recordar que fue el Presidente estadounidense J.F. Kennedy, en la década de los 60, quien lanzó ante el Congreso de Estados Unidos la necesidad de proteger los derechos de los consumidores, lo que hizo a través de su ya célebre frase: consumers, by definition, include all us.

Cuando se menciona esta interesante figura legal se advierte que uno de sus aspectos fundamentales se encuentra en el concepto de fluid recovery, que se pudiera traducir como "reparación fluida". Si la composición del grupo no puede definirse con precisión porque las personas que lo integran ni son identificadas ni son posiblemente identificables, el juez decreta una medida que alcanza a un "género" de interesados (de Llera Suárez, 1993; de Ángel Yágüez, 1994).

Cada vez que nos hemos encontrado en el marco del Derecho español con cuestiones que han afectado a una pluralidad de grupos, y han abarcado múltiples intereses, nos hemos topado, al mismo tiempo, con enormes dificultades en cuanto a una solución satisfactoria de las mismas se refiere. Esto ha ocurrido, verbi gratia, entre otros muchos casos, en relación al aceite de colza desnaturalizado, respecto del síndrome de Jacobs (vacas locas), la presa de Tous, la catástrofe de Doñana, la talidomida, el problema de las participaciones preferentes, las cláusulas suelo, el fraude por las emisiones contaminantes de automóviles Volkswagen, lo que viene a representar un panorama de innumerables reclamaciones, todas ellas, muy lejanas de encontrar soluciones satisfactorias para todos los afectados.

En contraste con lo expuesto, en cambio, en el Derecho norteamericano su aplicación ha sido materializada con bastante éxito en repetidas ocasiones. Así, ha acontecido en lo que afecta al amianto, como en el litigio Manville mencionado supra, a las prótesis mamarias de silicona, en el pleito Dow Chemical, en el que las demandas se concentraron en el

\footnotetext{
${ }^{21}$ Ver Gestión Práctica de Riesgos Laborales. Jurisprudencia, págs. 30 y ss, no 73, julio-agosto 2010); Poyatos Matas, G. (2015). La Justicia y el amianto, crónica de un asesino silencioso. Huffpost. Recuperado de: http://www.huffingtonpost.es/gloria-poyatos-matas/ la-justicia-y-el-amianto-_b_7841184.html ; Redacción de Protección Laboral, Bienestar Laboral, blog, enfermedades profesionales, 1 de marzo de 2016
} 
Estado de Alabama, a los efectos nocivos del tabaco, cuyas resoluciones se certificaron en el Estado de California y, más recientemente, respecto de la falta, por parte de la citada compañía alemana, de soluciones técnicas para el control de emisiones de óxidos de nitrógeno exigidas en Estados Unidos para dichos vehículos, entabladas, por ejemplo, en el Estado de California.

En nuestra posición de derecho comparado, por consiguiente, aconsejamos su pronta implantación en nuestro país, a sabiendas de que nuestro ordenamiento jurídico ha optado por la defensa individualizada de los derechos de las personas, tradicionalmente, debido a la influencia del derecho romano sobre la legislación, jurisprudencia y doctrina prevalentes en el derecho continental. Lógicamente, en un sistema judicial como el imperante en el derecho estadounidense, es el juez quien "certifica" la determinación inicial en torno a la propia existencia de la "clase", antes de proceder a la admisión de la acción, que permita entablar el respectivo proceso. Así queda recogido en la Regla 23 (c) (1). Por consiguiente, el Juez tiene la potestad de estimar que sólo un sector o "subclase" de los componentes de dicha "clase" reúne los requisitos para pleitear por esta vía legal. Es obvio que si la litis continúa, la sentencia no producirá los efectos de res iudicata respecto de los miembros excluidos, que tendrán abierto el recurso al proceso ordinario en orden a litigar la cuestión controvertida, con el derecho a apelar la decisión judicial si encuentran motivos de fondo contrarios a sus intereses en el fallo del tribunal.

Desde un punto de vista socioeconómico, el cambio de mayor calado en el derecho se produjo con la progresiva aparición y consolidación de la sociedad de consumo, a partir de la revolución industrial, y de la introducción de los sistemas de "producción en masa" de los bienes de consumo, que abrieron la puerta a su comercialización y "distribución en masa", y su consumo por "masas de personas" componentes de la mencionada sociedad de consumo. Una vez admitida la conveniencia del recurso a las condiciones generales de la contratación (standard form contracts), en sus múltiples facetas (verbi gratia, contratos-formulario, contratos tipo, contratos de masas, contratos estándar) se vería que no es viable, en términos generales, la negociación de estipulaciones concretas en el actual tráfico de masas, ya que ello conduciría, inexorablemente, al incremento del coste de los productos por contradecir la teoría del bienestar social. La incógnita a resolver estriba en dar con el mecanismo adecuado para obtener un reparto eficaz del riesgo contractual, que debemos atribuirlo a quien con un menor gasto puede controlarlo (cheapest cost avoider), en orden a lograr una eficiencia distributiva y conseguir un mejor reparto de los recursos.

Además, el creciente y continuo desarrollo del derecho común extracontractual (tort law), ante la constante y abundante proliferación de los contratos de adhesión, hace necesaria la aparición de nuevos remedios en el ámbito de la responsabilidad aquiliana (verbi gratia, the tort of unconscionability), máxime teniendo en cuenta que en el mundo actual aproximadamente el $99 \%$ de los contratos que se realizan en la práctica cotidiana 
son estándar, puesto que el contratante no tiene más opción que la de subscribirlos. La importancia de dicha doctrina en el contexto de un contrato de adhesión estriba en el hecho de que si sus cláusulas pudieran ser calificadas de injustas, abusivas o leoninas, se acepta su revisión, para lo que en el derecho norteamericano se recurre al mentado tort, que aparece codificado en el Código de Comercio Uniforme y que, aunque carece de definición, con su enorme componente de ética y moralidad que apela a sentimientos de justicia, favorece en un buen número de litigios a la parte más débil de la contratación ${ }^{22}$ Su alcance legal pudiera ser en la práctica substancialmente coincidente con la función que desempeña al principio general de buena fe en el marco del Derecho español. ${ }^{23}$

\section{CONCLUSIONES}

Como hemos mencionado a lo largo de este artículo, en virtud de los compromisos internacionales como son los SDGs, los Estados se comprometieron inter alia a la protección de la dignidad, a eliminar todas formas de discriminación, a garantizar la equidad y el estado de derecho y, finalmente, a proteger el medio ambiente. Por tanto, estarían obligados a asegurar el pleno ejercicio de los derechos fundamentales a través su acceso universal, por lo menos un nivel básico, así como el acceso a los recursos cuando tales derechos sean vulnerados por el sector público o el sector privados.

Por consiguiente, en un mundo caracterizado por unequal legal battles entre el sector privado y los afectados por las violaciones de los Derechos Humanos y el medio ambiente, somos de la opinión de que la vía para solventar los obstáculos de naturaleza económica que implican juicios civiles contra empresas multinacionales, es la de implementar el sistema de acciones de defensa de los intereses colectivos, como el caso de la ley 20161547, en Francia.

De hecho, en las últimas décadas asistimos a una creciente proliferación de los contratos en masa, lo que propicia la aparición de daños en masa que requieren el recurso a las acciones de defensa de intereses colectivos, que permiten un acceso a la justicia, del que se carece en el marco legal europeo y dentro del organigrama judicial español, en la actualidad, que es lo que más nos ocupa y preocupa. Todo ello con el objetivo imperante de potenciar el desarrollo sostenible, como uno de los mejores legados que podemos dejar a nuestras futuras generaciones.

\footnotetext{
${ }^{22}$ LawInfo. (s.f) Un panorama del Código de Comercio Uniforme. Recuperado de: http://abogados.lawinfo.com/recursos/ley-denegocio/un-panorama-del-c-digo-de-comercio-uniforme.html

${ }^{23}$ Entre otros muchos, podemos citar los contratos de aparcamiento de vehículos, entradas a espectáculos públicos, resguardos de mercancías depositadas en consignas y almacenes, justificantes de pago de compras en grandes superficies, comprobantes del abono con visa de las prestaciones en estaciones de servicio, etc. Véase: Slawson, W. D. (1971). Standard form contracts and democratic control of lawmaking power. Harvard Law Review, 529-566; Viguri, A. (2007). Los contratos comerciales internacionales: análisis de la compraventa desde la perspectiva del derecho comparado: derecho español, derecho norteamericano, principios Unidroit y Convención de Viena. Colegio de Registradores de la Propiedad y Mercantiles de España.
} 


\section{REFERENCIAS BIBLIOGRÁFICAS}

Asamblea Nacional. (18 de noviembre del 2016). Para modernizar la justicia del Siglo XXI. [Ley 2016-1547]. Recuperado de: https://www.legifrance.gouv.fr/eli/ loi/2016/11/18/JUSX1515639L/jo

A. Azagra y Marian Gili. (2005). Guia InDret de jurisprudencia sobre responsabilidad civil por daños del amianto. Indret (2).Recuperado de: http://www.asviamie.org/ documentos/condenas_amianto.pdf

Bonfanti, A. (2012). Imprese multinazionali, diritti umani e ambiente: profili di diritto internazionale pubblico e privato (Vol. 18). Milán: Giuffrè Editore.

Bravo, A. A. S. (2013). Injusticia ambiental y derecho humano al agua. Justicia y medio ambiente, 151-170. Punto Rojo.

Cernic, J. L. (2013). Obligaciones de las empresas en el marco del derecho humano al medio ambiente sano y al agua. La responsabilidad de las multinacionales por violaciones de derechos humanos (pp. 193-213). Servicio de Publicaciones.

Čiegis, R., y Ramanauskienè,J. (2009). Sustainable developmentand itsassessment. Applied Economics: Systematic Research, 3(2). Recuperado de: http://ejournals.vdu.It/ index.php/applied-economics/article/view/429.

Código Civil Español [Código]. (1889) Recuperado de: http://civil.udg.es/normacivil/ estatal/CC/RD25071889.htm

Comisión de las Comunidades Europeas. (26 de julio de 1999). Por la que se adapta al progreso técnico por sexta vez el anexo I de la Directiva 76/769/CEE del Consejo relativa a la aproximación de las disposiciones legales, reglamentarias y administrativas de los Estados miembros que limitan la comercialización y el uso de determinadas sustancias y preparados peligrosos (amianto). [Directiva 1999/77/CE]. Recuperado de: http://eur-lex.europa.eu/LexUriServ/LexUriServ. do?uri=CELEX:31999L0077:ES:HTML

Consejo de la Unión Europea. (9 de octubre de 1978). Fusiones nacionales de sociedades anónimas: tercera directiva. [Tercera Directiva 78/855/CEE]. Recuperado de: http:// eur-lex.europa.eu/legal-content/ES/TXT/?uri=LEGISSUM:I26006

- (19 de septiembre de 1983). Sobre la protección de los trabajadores contra los riesgos relacionados con la exposición al amianto durante el trabajo (segunda Directiva particular con arreglo al artículo 8 de la Directiva 80/1107/CEE). 
[Directiva 83/477/CEE]. Recuperado de: http://www.insht.es/portal/site/Insht/ menuitem.1f1a3bc79ab34c578c2e8884060961ca/?vgnextoid=5b837118e47 66110VgnVCM100000dc0ca8c0RCRD\&vgnextchannel=75164a7f8a651110 VgnVCM100000dc0ca8c0RCRD

- (25 de junio de 1991). Por la que se modifica la Directiva 83/477/CEE sobre la protección de los trabajadores contra los riesgos relacionados con la exposición al amianto durante el trabajo (segunda directiva particular con arreglo al artículo 8 de la Directiva 80/1107/CEE). [Directiva DIRECTIVA 91/382/CEE]. Recuperado de: http://www.insht.es/portal/site/Insht/ menuitem.1f1a3bc79ab34c578c2e8884060961ca/?vgnextoid=baf50399641972 10VgnVCM1000008130110aRCRD\&vgnextchannel=ff3cc6b33a9f1110V gnVCM100000dc0ca8c0RCRD

- (7 de abril de 1998). Relativa a la protección de la salud y la seguridad de los trabajadores contra los riesgos relacionados con los agentes químicos durante el trabajo (decimocuarta Directiva específica con arreglo al apartado 1 del artículo 16 de la Directiva 89/391/CEE). [DIRECTIVA 98/24/CE]. Recuperado de: http://www.insht.es/portal/site/Insht/ menuitem.1f1a3bc79ab34c578c2e8884060961ca/?vgnextoid=412dfa94e2c86110 VgnVCM $100000 d c 0 c a 8 c 0 R C R D \& v g n e x t c h a n n e l=75164 a 7 f 8 a 651110$ VgnVCM100000dc0ca8c0RCRD

- (27 de marzo del 2003). Por la que se modifica la Directiva 83/477/CEE del Consejo sobre la protección de los trabajadores contra los riesgos relacionados con la exposición al amianto durante el trabajo. [Directiva 2003/18/CE]. Recuperado de: http://www.insht.es/portal/site/Insht/ menuitem.1f1a3bc79ab34c578c2e8884060961ca/?vgnextoid=23760399641972 $10 \mathrm{VgnVCM} 1000008130110 a R C R D \& v g n e x t c h a n n e l=f f 3 c c 6 b 33 a 9 f 11$ 10VgnVCM100000dc0ca8c0RCRD

. (20 de junio del 2007). Por la que se modifica la Directiva 89/391/CEE del Consejo, sus directivas específicas ylas Directivas 83/477/CEE, 91/383/CEE, 92/29/CEE y 94/33/CE del Consejo, a fin de simplificar y racionalizar los informes sobre su aplicación práctica. [Directiva 2007/30/CE]. Recuperado de: http://www.insht.es/portal/site/Insht/ menuitem.1f1a3bc79ab34c578c2e8884060961ca/?vgnextoid=4e9f72b6025fe310 VgnVCM $1000008130110 a R C R D \& v g n e x t c h a n n e l=f f 3 c c 6 b 33 a 9 f 1110$ VgnVCM100000dc0ca8c0RCRD

- (5 de abril del 2011). Fusiones de las sociedades anónimas. [Directiva 2011/35/UE]. Recuperado de: http://eur-lex.europa.eu/LexUriServ/ LexUriServ.do?uri=OJ:L:2011:110:0001:0011:es:PDF 
- (26 de febrero del 2014). Por la que se modifican las Directivas 92/58/CEE, 92/85/CEE, 94/33/CE, 98/24/CE del Consejo y la Directiva 2004/37/CE del Parlamento Europeo y del Consejo, a fin de adaptarlas al Reglamento (CE) $n$ o 1272/2008 sobre clasificación, etiquetado y envasado de sustancias y mezclas. [Directiva 2014/27/UE]. Recuperado de: http://www.insht.es/portal/site/Insht/ menuitem.1f1a3bc79ab34c578c2e8884060961ca/?vgnextoid=52c39bac53c9441

OVgnVCM $1000008130110 a R C R D \& v g n e x t c h a n n e l=f f 3 c c 6 b 33 a 9 f 111$ OVgnVCM100000dc0ca8c0RCRD

Corte Suprema de California. (mayo 10 de 1971). Vasquez v. Superior Court, 484 P.2d 964. Recuperado de: https://www.courtlistener.com/opinion/1185687/vasquez-vsuperior-court/

Cortina, A. (2006). Ética Del Desarrollo: Un Camino Hacia La Paz. Revista De Ciencias Sociales (192), 3-18.

Cyran, O. (2013). En Bangladesh, los asesinos del" prêt-a-porter". Le Monde diplomatique en español, (212), 1-23.

Dallo, J. (2016). Una Agenda de Desarrollo Sostenible para un nuevo ecosistema mundial. Temas para el debate, (254), 41-43.

De Ángel Yágüez, R. (1994). La posición del consumidor y el ejercicio de sus derechos: Daños causados por productos defectuosos. Estudios sobre el Derecho de consumo (pp. 58-107). Fundación Iberdrola.

De Llera Suárez-Bárcena, E. (1993). El Ministerio Fiscal y los consumidores. La Ley: Revista jurídica española de doctrina, jurisprudencia y bibliografía, (3), 795-801.

De Miguel Beriain, I. (2013). Algunas reflexiones acerca de la tragedia del rana plaza:¿ quién fue el responsable?. Dilemata, (13), 121-152.

Dernbach, J. C., y Cheever, F. (2015). Sustainable Development and Its Discontents. Transnational Environmental Law, 4(02), 247-287.

Evans, B. A. (2014). Accord on Fire and Building Safety in Bangladesh: An International Response to Bangladesh Labor Conditions. North Carolina Journal of International Law and Commercial Regulation (40), 597-626.

Federal Register. (s.f). Code of federal regulations (annual edition). U. S. Government publishing office. Recuperado de: https://www.gpo.gov/fdsys/browse/collectionCfr. action?collectionCode=CFR 
Foundation for Democracy and Sustainable Development. (s.f). UN Sustainable DevelopmentGoal(SDG)16-importance of participatoryinstitutions \& policymaking. Recuperado de: http://www.fdsd.org/ideas/sustainable-development-goal-sdg16-democratic-institutions/

Garrido, L. (2009). Una dura condena da esperanzas a los afectados por el amianto. El País. Recuperado de: http://elpais.com/diario/2009/08/17/ sociedad/1250460003_850215.html

Gomes, W. (2013). Reason and Responsibility: the Rana Plaza Collapse. openDemocracy. Recuperado de: https://www.opendemocracy.net/opensecurity/william-gomes/ reason-and-responsibility-rana-plaza-collapse

Guimarães, R. P. (2015). Desarrollo sustentable:¿ todavía esperando a Godot? TERRA: Revista de Desarrollo Local, (1), 67-98.

i LLobet, J. V., y Manzano, J. J. (2007). El derecho a un medio ambiente sano: su reconocimiento en el constitucionalismo comparado y en el derecho internacional. Teoría y realidad constitucional, (20), 513-533.

Kim, R. E. (2016). The Nexus between International Law and the Sustainable Development Goals. Review of European, Comparative \& International Environmental Law, 25(1), 15-26.

Krajnc, D., y Glavič, P. (2005). A model for integrated assessment of sustainable development. Resources, Conservation and Recycling, 43(2), 189-208.

Laronze, F., y de Quénaudon, R. (2013). Réflexions juridiques après la tragédie du Rana Plaza. Revue de droit du travail, (7), 487-490.

LawInfo. (s.f) Un panorama del Código de Comercio Uniforme. Recuperado de: http:// abogados.lawinfo.com/recursos/ley-de-negocio/un-panorama-del-c-digo-decomercio-uniforme.html

Lehtonen, M. (2004). The environmental-social interface of sustainable development: capabilities, social capital, institutions. Ecological economics, 49(2), 199-214.

Lund-Thomsen, P., y Lindgreen, A. (2014). Corporate social responsibility in global value chains: Where are we now and where are we going?. Journal of Business Ethics, 123(1), 11-22.

Marullo, M. C. (2015). La lucha contra la impunidad: el Foro Necessitatis. Indret: Revista para el Análisis del Derecho, (3), 9-47. 
Marullo, Mạ. Ch. y Pallares, L. (junio de 2016). El acceso a la justicia y la responsabilidad de las multinacionales por las violaciones a los derechos humanos: reflexiones sobre la aportación de los SDGs. En VI Encuentro luso-español de profesores de Derecho Internacional y relaciones internacionales. Colegio Mayor Rector Peset. Plaza del Horno de San Nicolás, 4.

Marullo, M. C., y Zamora Cabot, F. J. (2016). Transnational human rights litigations: Kiobel's touch and concern: A test under construction. Papeles El tiempo de los derechos (1), 1-38. Recuperado de: http://repositori.uji.es/xmlui/bitstream/ handle/10234/159819/72099.pdf?sequence=1\&isAllowed=y

Marzá, D. G. (2009). ¿Agentes de justicia?: La responsabilidad social de las empresas como factor de desarrollo. In Pobreza y libertad: erradicar la pobreza desde el enfoque de las capacidades de Amartya Sen. Madrid, Tecno, 193-210.

Mendizábal, T. M. (2013). Nacimiento y evolución del concepto de desarrollo sostenible. ¿'Es sostenible el mundo en que vivimos?, 33-51.

Mesa, M. (2016). El Objetivo n. o 16 de Desarrollo Sostenible: paz, seguridad y gobernanza. Temas para el debate, (254), 37-40.

Ministerio de la Presidencia. (29 de octubre del 2004). Por el que se aprueba el texto refundido de la Ley sobre responsabilidad civil y seguro en la circulación de vehículos a motor. [Real Decreto Legislativo 8/2004]. Recuperado de: http://noticias.juridicas. com/base_datos/Privado/rdleg8-2004.html

- (7 de diciembre del 2001). ORDEN por la que se modifica el anexo I del Real Decreto 1406/1989, de 10 de noviembre, por el que se imponen limitaciones a la comercialización y al uso de ciertas sustancias y preparados peligrosos. Recuperado de: http://www.boe.es/boe/dias/2001/12/14/pdfs/A47156-47157.pdf

Naciones Unidas. (19 diciembre 1983). Proceso de elaboración de la perspectiva ambiental hasta el Año 2000 y más adelante. [Resolución A/RES/38/161].

_. (4 de agosto de 1987). Desarrollo y cooperación económica internacional: medio ambiente. Informe de la Comisión Mundial sobre el Medio Ambiente y el Desarrollo. [A/42/427].

_. (25 de septiembre del 2015). Transformar nuestro mundo: la Agenda 2030 para el Desarrollo Sostenible. [Resolución aprobada. Documento A/RES/70/1].

_. (s.f). 17 objetivos para transformar nuestro mundo. Recuperado de: http://www. un.org/sustainabledevelopment/es/ 
- (s.f). La Agenda de Desarrollo Sostenible. Recuperado de: http://www.un.org/ sustainabledevelopment/es/la-agenda-de-desarrollo-sostenible/

_. Objetivo 16: Promover sociedades, justas, pacíficas e inclusivas. Recuperado de: http://www.un.org/sustainabledevelopment/es/peace-justice/

Pigrau, A. (2014). The Texaco-Chevron case in Ecuador: Law and justice in the age of globalization. Revista Catalana de Dret Ambiental, 5(1), 1-44.

Poyatos Matas, G. (2015). La Justicia y el amianto, crónica de un asesino silencioso. Huffpost. Recuperado de: http://www.huffingtonpost.es/gloria-poyatos-matas/lajusticia-y-el-amianto-_b_7841184.html

Pozzi, S. (2016). 10 empresas más grandes que 180 países. Recuperado de: http://economia. elpais.com/economia/2016/09/29/actualidad/1475150102_454818.html

Redclift, M. (1991). The multiple dimensions of sustainable development. Geography, Vol.76 (1), 36-42.

Ruiz, J. J., y Daudí, M. C. (2014). La protección del medio ambiente en el ámbito internacional y en la Unión Europea. Valencia, España: Tirant lo blanch.

Sachs, J. D. (2015). The age of sustainable development. Columbia University Press.

Sánchez, J. M. E. (2016). Globalidad y solidaridad desde la perspectiva medioambiental. La Albolafia: Revista de Humanidades y Cultura, (6), 133-166.

Simkiss, D. (2015). The millennium development goals are dead; long live the sustainable development goals. Journal of Tropical Pediatrics 61 (4): 235-237.

Slawson, W. D. (1971). Standard form contracts and democratic control of lawmaking power. Harvard Law Review, 529-566.

Solanes, A. (2016). ¿Empresas sin escrúpulos? Sobre la responsabilidad social corporativa. Alrevésyalderecho. Recuperado de: http://alrevesyalderecho.infolibre.es/?p=4415.

Skinner, G., McCorquodale, R., y De Schutter, O. (2013). The Third Pillar: Access to Judicial Remedies for Human Rights Violations by Transnational Business. ICAR/CORE/ECCJ. Recuperado de: http://corporatejustice.org/documents/publications/eccj/the third_pillar_-access_to_judicial_remedies_for_human_rights_violation.-1-2.pdf

Supreme Court. (1937). Federal Rules of Civil Procedure. Recuperado de: https://www. law.cornell.edu/rules/frcp\#Form_6_1_target 
Tribunal de Justicia de la Unión Europea, Sala Quinta. (5 de marzo de 2015). Sentencia. Asunto C-343/13. [MP C. Vajda].

Tribunal Superior de Justicia del País Vasco, Sala de lo Social. (25 de octubre del 2005). Sentencia Social № 2492/2005. Sección 1, Rec 1387/2005. [MP Manuel Díaz De Rabago Villar].

_. Sala de lo Social. Sentencia 15/15/2014. Recurso 1263/2014.

Tribunal Supremo, Sala Penal. (30 de noviembre de 1990). Núm. 3.851.-Sentencia. [MP José Antonio Martin Pallin].

. Sala de lo social. (30 de junio de 2010). Sentencia. Rec $n^{\circ} 4123 / 20008$. [MP Luis Fernando de Castro Fernández].

_. Sala de lo social. (23 de marzo del 2015). STS 2103/2015. [MP Luis Fernando de Castro Fernández].

U.S. Court of Appeals for the Fifth Circuit. (1973). Borel v. Fibreboard. 493 F.2d 1076 (5th Cir. 1973) Recuperado de: http://law.justia.com/cases/federal/appellate-courts/ F2/493/1076/4552/

. (1991). Corrosion Proof Fittings, et al., Petitioners, v. the Environmental Protection Agency and William K. Reilly,administrator, Respondents, 947 F.2d 1201 (5th Cir. 1991) Recuperado de: http://law.justia.com/cases/federal/appellate-courts/ F2/947/1201/153685/

U.S. District Court for the Southern District of New York. (27 de junio de 1991). In re Joint Eastern and Southern District Asbestos Litigation. 129 B.R. 710 (S.D.N.Y. 1991) Recuperado de: http://law.justia.com/cases/federal/district-courts/ BR/129/710/1871302/

Viguri, A. (2007). Los contratos comerciales internacionales: análisis de la compraventa desde la perspectiva del derecho comparado: derecho español, derecho norteamericano, principios Unidroit y Convención de Viena. Colegio de Registradores de la Propiedad y Mercantiles de España.

Zamora Cabot, F. J. (2015). Desarrollo sostenible y empresas multinacionales: Un estudio sobre los acaparamientos de tierra (land grabbings) en clave de responsabilidad. Papeles El tiempo de los derechos (4), 1-24. Recuperado de: http://repositori.uji.es/ xmlui/bitstream/handle/10234/159489/Desarrollo\%20Sostenible_Zamora_2015. pdf?sequence=1\&isAllowed=y 